\title{
Comparison of Long-Term Efficacy of MIS-TLIF Intraoperative Implants in Patients with Osteoporosis
}

\author{
Yao Li, Shengfu Liu, Zhimin He $\mathbb{D}$, and Shunzhi Yu \\ Department of Orthopedics, Shanghai Tenth People's Hospital, Tongji University School of Medicine, Shanghai, China \\ Correspondence should be addressed to Zhimin He; hezhimin301@163.com and Shunzhi Yu; maotoutou@163.com
}

Received 19 January 2022; Revised 26 January 2022; Accepted 8 February 2022; Published 27 February 2022

Academic Editor: Min Tang

Copyright (c) 2022 Yao Li et al. This is an open access article distributed under the Creative Commons Attribution License, which permits unrestricted use, distribution, and reproduction in any medium, provided the original work is properly cited.

\begin{abstract}
Osteoporosis and degenerative spinal disease are still an unsolvable surgical problem. It is still difficult to solve the complications related to postoperative osteoporosis, such as cage subsidence, displacement, and retraction. Expandable interbody cage is a recent innovation and an increasingly popular alternative to standard static cage. However, the clinical efficacy of MIS-TLIF combined with expandable cage for the treatment of osteoporosis has limited reports. The purpose of this paper was to analyze the efficacy of MIS-TLIF with expandable cage in patients with degenerative lumbar disease with osteoporosis. Patients with osteoporosis who received single-level MIS-TLIF and were followed up for at least 1 year were included. The outcome measures are as follows: clinical features, perioperative period, and neurological complications. JOA score and VAS pain score were used to analyze the improvement of patients' function. Imaging analysis included segmental lordosis (SL), lumbar lordosis (LL), intervertebral disc height $(\mathrm{DH})$, and the ratio of cage height to preoperative $\mathrm{DH}(\mathrm{RCD})$. The final data analysis included 284 patients with osteoporosis. 178 patients used static cages, and 106 patients used expandable cages. There was no significant difference in baseline characteristics, surgical indexes, and JOA and VAS scores between the two groups. There was no difference in SL or LL between static group and expandable group. There was no significant difference in preoperative DH between the two groups. The RCD in the expansion group was significantly lower than that in the static group. The intraoperative and postoperative sedimentation rate in the static group was significantly higher than that in the expandable group. The use of expandable cages in MIS-TLIF has shown good results for the treatment of degenerative lumbar diseases with osteoporosis. Through appropriate surgical techniques, the expandable cage can reduce the risk of cage sinking.
\end{abstract}

\section{Introduction}

Osteoporosis is a degenerative disease with bone loss and structural deterioration. It is the most common challenge of lumbar interbody fusion. Although minimally invasive transforaminal lumbar interbody fusion (MIS-TLIF) has been widely used to treat patients with osteoporosis, it is still difficult to solve the complications related to interbody fusion cage, such as interbody fusion cage sinking, displacement, and retraction [1-3]. Osteoporosis and degenerative spinal disease remain an unsolvable surgical problem and increase uncertainty.

At present, the interbody fusion cage that can be used for interbody fusion can be divided into static fusion cage and expandable fusion cage. The expandable interbody cage is a latest innovation designed to increase the adjustability of cage height and improve the convenience of insertion [4]. MISTLIF technology has been developed to include expandable intervertebral devices, which has the potential advantages of better disc height recovery and segmental lordosis. Although expandable cages have been widely used in a variety of spinal diseases, there are few reports on the use of MIS-TLIF and expandable cages in patients with osteoporosis.

Endplate damage is closely related to cage sinking and movement. There are two types of cage sinking, one is caused by intraoperative endplate injury, and the other is gradually occurring after operation. The etiology of these two types of cage settlement should be considered separately [5]. One is gradually occurring after operation (self-hair), and the other is intraoperative endplate injury (iatrogenic). The former is 
the spontaneous reaction between cage and endplate. The latter is the violation of TCM-derived endplate during endplate preparation or stent insertion. For patients with osteoporosis, endplate injury usually occurs during stent implantation. Insertion of a relatively high cage into the narrow intervertebral space and posterior forward traction may increase the risk of endplate injury. Endplate damage may change the direction of the cage and increase the risk of cage sinking and movement. The expandable cage is inserted into the intervertebral disc space in a planar form and then expanded in situ to restore the intervertebral disc height [6]. Less insertion resistance and a uniform bottom-up expansion procedure may help to reduce the risk of endplate damage.

The purpose of this study was to analyze the efficacy of MIS-TLIF plus expandable cage in the treatment of patients with lumbar spongiform arthropathy or lumbar discitis who may be accompanied by osteoporosis. We hypothesized that this cage design can improve the safety of cage insertion and expansion while reducing the risk of cage complications.

\section{Methods}

2.1. Patient. A multicenter retrospective review was conducted of prospectively collected data from osteoporosis patients (the focus of this study is degenerative lumbar disease, so trauma patients were excluded. Also, due to the impaired lumbar spine, the fusion rate may be affected by lumbar trauma) treated with MIS-TLIF from 2012 to 2019. For this survey, our analysis was limited to primary intervention. Patients were classified according to cage type (static and expandable). Both static cage and expandable cage are forward convex cage. The degree of lordosis in the cage was selected according to the surgeon's preference. The $T$-score of all included patients was $<-2.5$ preoperative bone mineral density SD (osteoporosis). Inclusion in the analysis requires at least 1 year of follow-up.

2.2. Surgical Technique. All patients underwent general tracheal anesthesia in prone position. The MIS-TLIF technology used has been described in literature $[7,8]$. Bone was collected as an autologous graft during facetectomy. Implant interbody fusion cage includes static and scalable design. For both operations, interbody implantation begins with endplate preparation. Then, the intervertebral disc is removed and the vertebral endplate is scraped off to expose the bleeding bone without fracture. After peeling the endplate, autologous bone was filled into the anterior intervertebral disc space. Subsequently, static interbody spacers or expandable interbody devices were filled with autologous bone. For static cages, place the instrument in the disc space. According to the judgment of the surgeon, the cage of appropriate size was selected during the operation. The cage height was selected after the intraoperative test to achieve the best traction effect. The cages used in this series are bullet shaped. All cages are radiopaque peek devices with radiopaque marks to determine the position of the cage. For the expandable interbody fusion cage, the interbody fusion cage is placed into the intervertebral disc space under fluoroscopy and then expanded to an appropriate height by using a T-shaped rotating handle with a torque limiting screwdriver head. The degree of expansion depends on the judgment of the surgeon in order to achieve the most appropriate expansion (Figure 1). Intraoperative fluoroscopy confirmed the placement of the cage. No patients were compressed after cage placement.

2.3. Data Collection and Outcome Measures. Baseline demographic data were collected, including age, gender, height, weight, body mass index, bone marrow density, age-adjusted Charlson comorbidity index (ACCI) [9], and American Society of Anesthesiologists (ASA) physical condition classification score. All subjects were invited to complete the clinical evaluation, and the data were obtained at 1, 3, and 6 months and every half a year until the end. The follow-up time was at least 12 months. The Japanese Orthopaedic Association (JOA) score was used to analyze the improvement of patients' clinical function. VAS pain score was measured to determine the severity of pain. Postoperative outcomes included medical and surgical complications, including adjacent segment disease, pseudoarthropathy, endplate injury, interbody fusion cage displacement, and subsidence. Cage settlement is evaluated by side pieces and is defined as $\geq 2 \mathrm{~mm}$ for the cage penetration of the endplate. When the fluoroscopy image determined that the endplate was in violation, the intraoperative subsidence was classified. The postoperative subsidence was classified according to the intraoperative fluoroscopy, but it appeared on the lateral film of 1-year follow-up. The definition of offset is that the horizontal offset measured by follow-up X-ray film is more than $2 \mathrm{~mm}$ compared with the X-ray film immediately after operation. Segmental lordosis (SL), lumbar lordosis (LL), and intervertebral disc height $(\mathrm{DH})$ were measured on anteroposterior and lateral X-rays obtained before and after operation. Lateral radiographic measurements were performed independently by three authors who did not perform the procedure. $\mathrm{DH}$ is defined as the distance between the midpoint of the upper endplate and the lower endplate. Cage/preoperative $\mathrm{DH}$ is the ratio of cage height to preoperative DH (RCD). SL is measured as the Cobb angle of the upper and lower endplates of the fusion layer. LL is measured as the transverse Cobb angle from L1 upper endplate to S1 upper endplate.

2.4. Statistical Analysis. Descriptive tests were used to retrospectively analyze the demographic and clinical characteristics of the entire patient cohort. The independent-sample Student $t$-test or Wilcoxon rank sum test was used to compare the continuous variables of the expandable cage group and static cage group. Categorical variables were compared by the chi-square test or Fisher exact test. SPSS 21.0 (IBM, Armonk, New York, USA) software was used for statistical evaluation. If the $P$ value is $<0.05$, the difference is considered significant.

2.5. Consequence. A total of 284 patients (178 static stents and 106 expandable stents) were included in the final analysis. No patients were lost to follow-up in this study, and all patients were operated in the abovementioned time frame. Baseline characteristics were similar in the static and expandable groups (Table 1). There was no significant difference in postoperative hospitalization, bed rest time, blood loss, and operation time between the two groups. These data are shown in Table 2. 


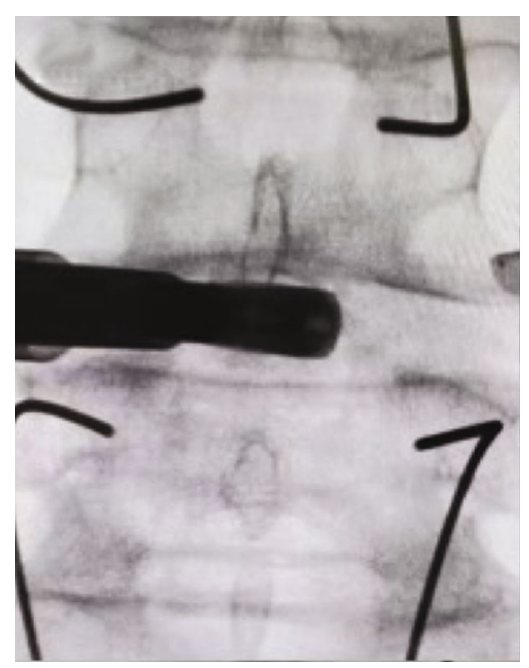

(a)

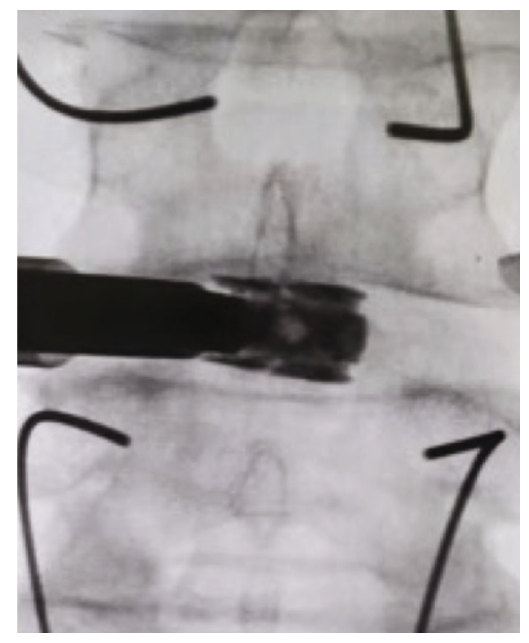

(c)

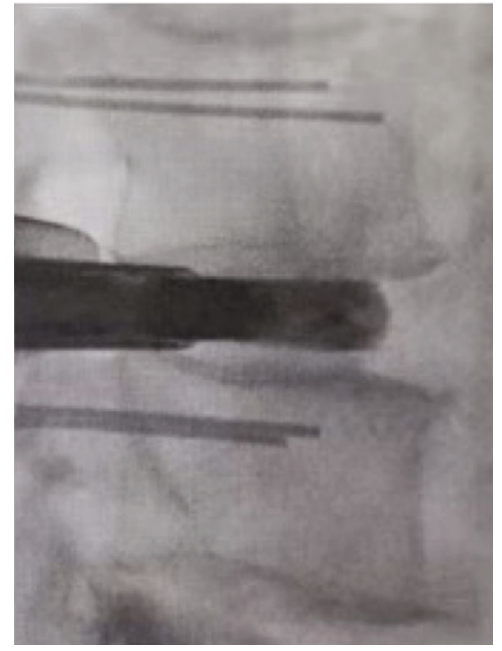

(b)

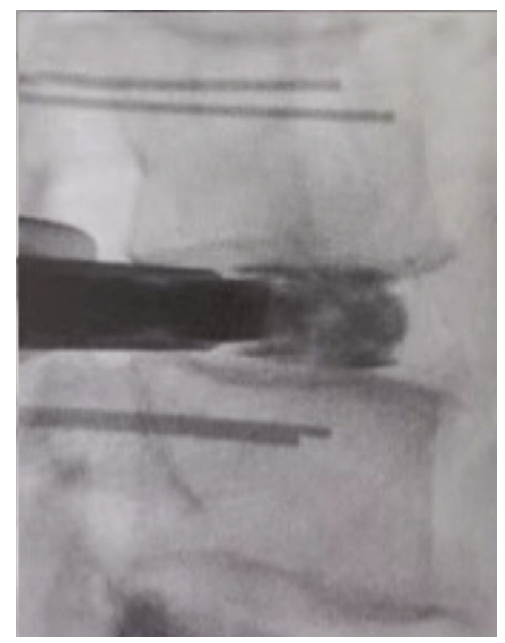

(d)

Figure 1: Expandable cage was placed into disc space in a flat form. Cage direction in the sagittal plane could be adjusted until the cage is parallel to the endplate before expansion $(a, b)$. Cage was expanded up to the appropriate height (c, d).

2.6. JOA and VAS Scores. All patients completed the selfreported questionnaire according to the instructions. The JOA score at 1 month, 3 months, 6 months, and 12 months of follow-up was significantly higher than that before operation $(P<0.05)$. No significant difference existed in the JOA score between the static group and expandable group. Compared with the preoperative VAS score, the VAS score of low back pain in both groups was significantly lower. There was no significant difference in the VAS score of low back pain between the static group and expandable group. Compared with the preoperative score, the VAS score at the leg time point was significantly lower in both groups. There was no significant difference in the VAS score of leg time point between the static group and expandable group. A detailed analysis of JOA and VAS scores is shown in Table 3 (Figure 2). Compared with the preoperative score, the JOA score and VAS score at 3 and 12 months of follow-up were significantly increased, and there was no significant difference between the static group and the expandable group.
2.7. Imaging Measurement and Perioperative Complications. There was no difference in SL or LL between the static group and expandable group. No difference existed in preoperative $\mathrm{DH}$ between the two groups. The RCD of the dilated group was significantly lower than that of the static group $(1.28 \pm 0.11$ vs. $1.62 \pm 0.28 ; P<0.01)$. The total incidence of intraoperative subsidence in the whole cohort was $12.0 \%$. The intraoperative subsidence rate in the static group was significantly more than that in the expansion group $(15.7 \%$ vs. $5.7 \% ; P=0.011$ ) (Figure 3). The total incidence of postoperative subsidence in the whole cohort was $9.5 \%$. The postoperative sedimentation rate in the static group was significantly more than that in the expandable group $(12.4 \%$ vs. $4.7 \% ; P$ $=0.034$ ) (Figure 4). The cage mobility of the whole cohort was $3.9 \%$. The cage mobility in the static group was significantly higher than that in the expandable group $(5.6 \%$ vs. $0.9 \% ; P=0.048)$. There was no significant difference between the two groups in dural incision, screw loosening, pseudoarthropathy, and adjacent segment diseases (Table 4). 
TABLE 1

\begin{tabular}{|c|c|c|c|}
\hline Characteristic & Static group $(N=178)$ & Expandable group $(N=106)$ & $P$ value \\
\hline Age (years) & $65.52 \pm 11.51$ & $64.27 \pm 11.31$ & 0.708 \\
\hline Sex (male : female) & $46: 132$ & $24: 82$ & 0.545 \\
\hline BMI & $25.02 \pm 3.37$ & $25.14 \pm 3.53$ & 0.570 \\
\hline CCI & $1.96 \pm 0.85$ & $1.83 \pm 0.78$ & 0.358 \\
\hline ASA & $2.47 \pm 1.13$ & $2.50 \pm 1.07$ & 0.226 \\
\hline Primary diagnosis & & & 0.102 \\
\hline Symptomatic lumbar disc herniation & 88 & 66 & \\
\hline Symptomatic lumbar spinal canal stenosis & 55 & 26 & \\
\hline Symptomatic lumbar spondylolisthesis & 35 & 14 & \\
\hline Operation level & & & 0.681 \\
\hline $\mathrm{L} 1 / 2$ & 10 & 4 & \\
\hline $\mathrm{L} 2 / 3$ & 18 & 7 & \\
\hline $\mathrm{L} 3 / 4$ & 35 & 18 & \\
\hline $\mathrm{L} 4 / 5$ & 66 & 45 & \\
\hline $\mathrm{L} 5 / \mathrm{S} 1$ & 49 & 32 & \\
\hline$T$-score & $1.68 \pm 0.40$ & $1.65 \pm 0.41$ & 0.751 \\
\hline Mean follow-up time (months) & $17.12 \pm 3.71$ & $17.81 \pm 3.87$ & 0.165 \\
\hline
\end{tabular}

Comparison of baseline patient characteristics between the expandable cage group and static cage group. * Statistically significant difference.

TABLe 2

\begin{tabular}{lccc}
\hline & Static group $(N=178)$ & Expandable group $(N=106)$ & $P$ value \\
\hline Operating time (min) & $121.73 \pm 22.76$ & $117.38 \pm 23.42$ & 0.579 \\
Intraoperative blood loss $(\mathrm{ml})$ & $132.74 \pm 29.04$ & $128.65 \pm 20.25$ & 0.203 \\
Bed rest time (days) & $3.43 \pm 1.08$ & $3.72 \pm 1.11$ & 0.812 \\
Hospitalization stay (days) & $5.43 \pm 1.07$ & $5.68 \pm 1.12$ & 0.553 \\
\hline
\end{tabular}

Comparison of surgical indicators between the expandable cage group and static cage group.

TABLE 3

\begin{tabular}{lccc}
\hline Parameter & Group & Baseline & 3 months \\
\hline \multirow{2}{*}{ JOA } & Static group & $17.04 \pm 4.50$ & $22.99 \pm 1.82$ \\
& Expandable group & $16.92 \pm 5.88$ & $23.26 \pm 1.93$ \\
\hline \multirow{2}{*}{ VAS (back) } & Static group & $5.07 \pm 2.66$ & $2.79 \pm 1.44$ \\
& Expandable group & $5.34 \pm 2.56$ & $3.09 \pm 1.43$ \\
\hline \multirow{2}{*}{ VAS (leg) } & Static group & $6.02 \pm 2.03$ & $3.03 \pm 1.45$ \\
& Expandable group & $6.00 \pm 1.87$ & $2.82 \pm 1.39$ \\
\hline
\end{tabular}

Comparison between the expandable cage group and static cage group regarding JOA and VAS scores.

\section{Discussion}

The incidence rate of osteoporosis in lumbar fusion is increasing as the number of elderly degenerative lumbar diseases increases. MIS-TLIF has been shown to improve intraoperative and early postoperative outcomes $[10,11]$. However, some studies have shown that patients with low lumbar BMD may have a relatively high incidence of interbody fusion cagerelated complications.

The correlation between subsidence and clinical outcomes still remains controversial. Many previous researches reported that subsidence may lead to poor clinical outcomes. For instance, the sinking of interbody fusion cage may lead to the gradual narrowing of the intervertebral disc height, which affects the anterior support of the spine, so as to prevent successful fusion, and lead to adverse results [12]. Osteoporosis is one of the important risk factors of cage subsidence previously reported. Previous studies have shown that bone mineral density is related to the stable failure load of endplate and cage $[13,14]$. Oh et al. found that the correlation between BMD and subsidence was very weak, and patients with score $<-3.0$ had an increased risk of subsidence [15]. Cho et al. found that the $T$-score of patients was $\leq-2.5$, and the sedimentation rate increased by $\geq-1.0$ compared with patients with $T$-score [16].

In recent years, more and more minimally invasive techniques have been used in spinal surgery, which has made 


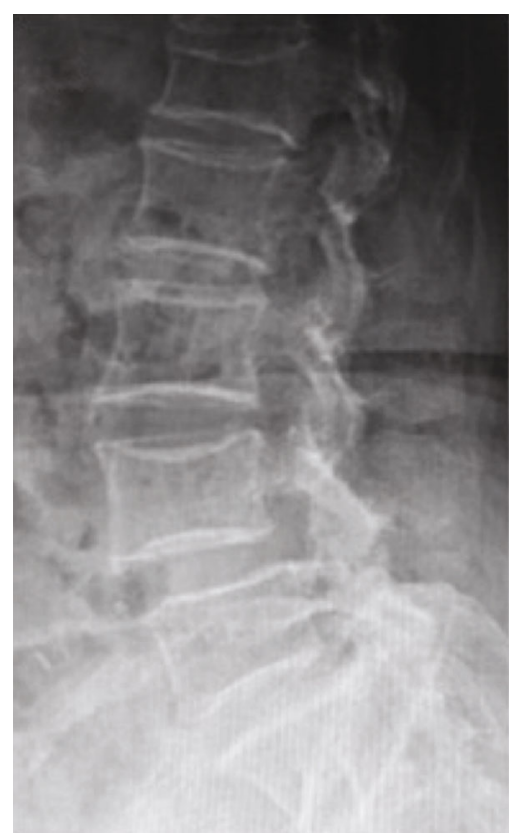

(a)

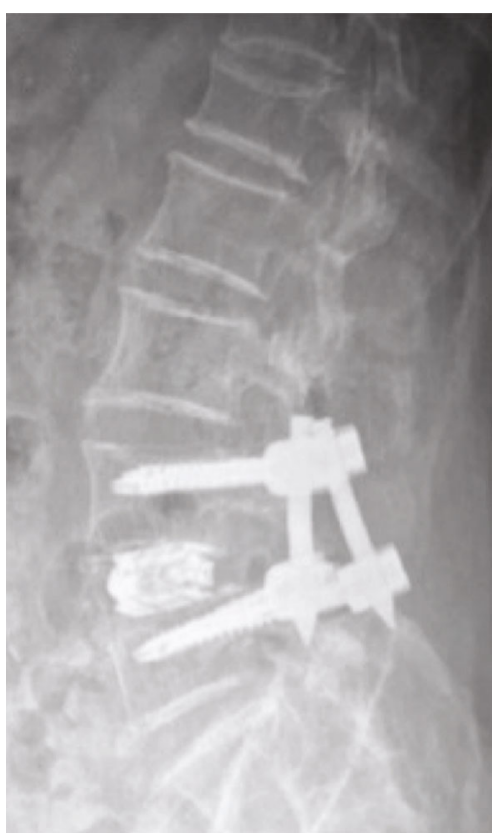

(b)

FIGURE 2: A spondylodiscitis case showing expandable cage without subsidence following MIS-TLIF: (a) preoperative lateral radiograph; (b) postoperative lateral radiograph.

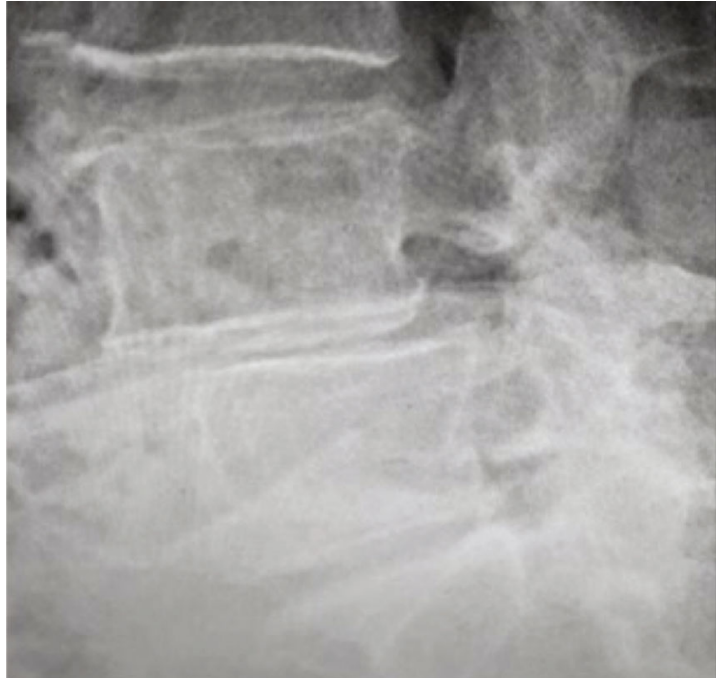

(a)

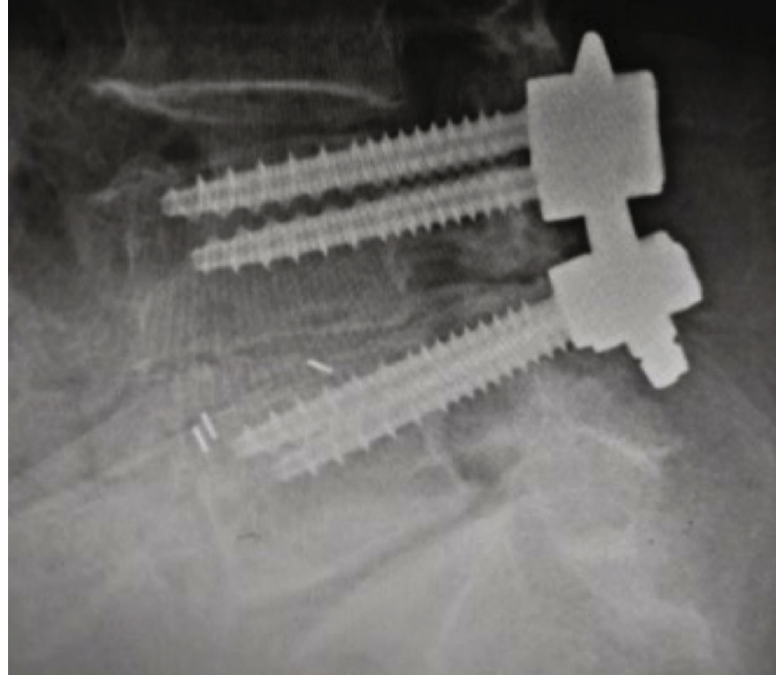

(b)

FIGURE 3: A case showing cage with intraoperative subsidence: (a) preoperative lateral radiograph; (b) immediately postoperative radiograph showing cage subsidence.

significant progress in the instruments of lumbar interbody fusion. The expandable ball cage is a relatively new invention, which assumes that the greater protrusion than the static ball cage is affected by extending the front column [17]. The expandable cage is designed to meet the challenge of MIS technology through oral approach. The aim is to reduce neurological complications by reducing retraction time, increasing insertion difficulty, and improving radiological results. The ability to deliver expandable interbody fusion cage through a narrow surgical cannula represents a significant progress in spinal surgery technology and meets the unmet clinical needs [6]. The expandable cage has shown good results in restoring intervertebral disc height and maintaining lordosis immediately after operation. Several studies support the view that expandable spacers provide greater improvements in disc height, recovery of segmental lordosis, foraminal decompression, and even patient-reported results compared with static cages [18-21]. However, some studies 


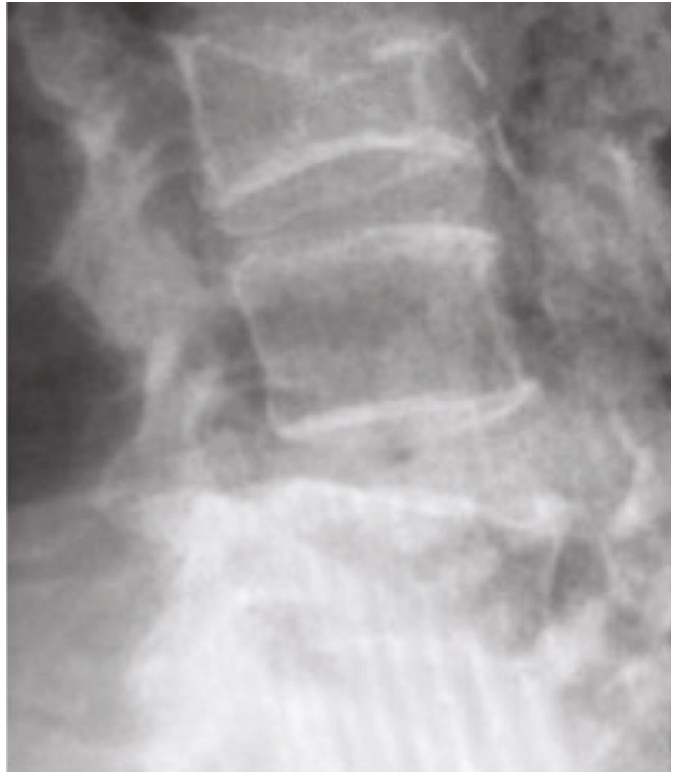

(a)

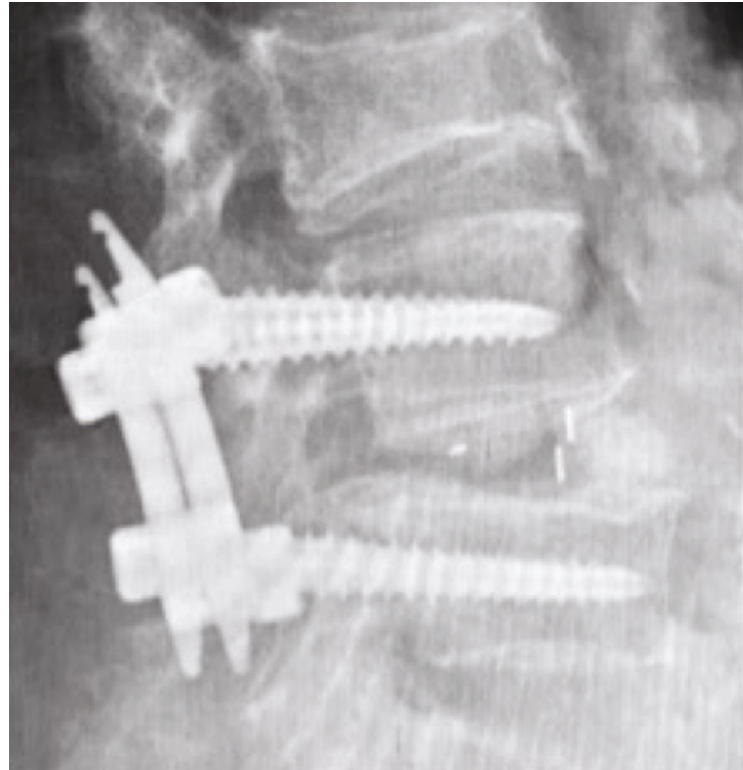

(b)

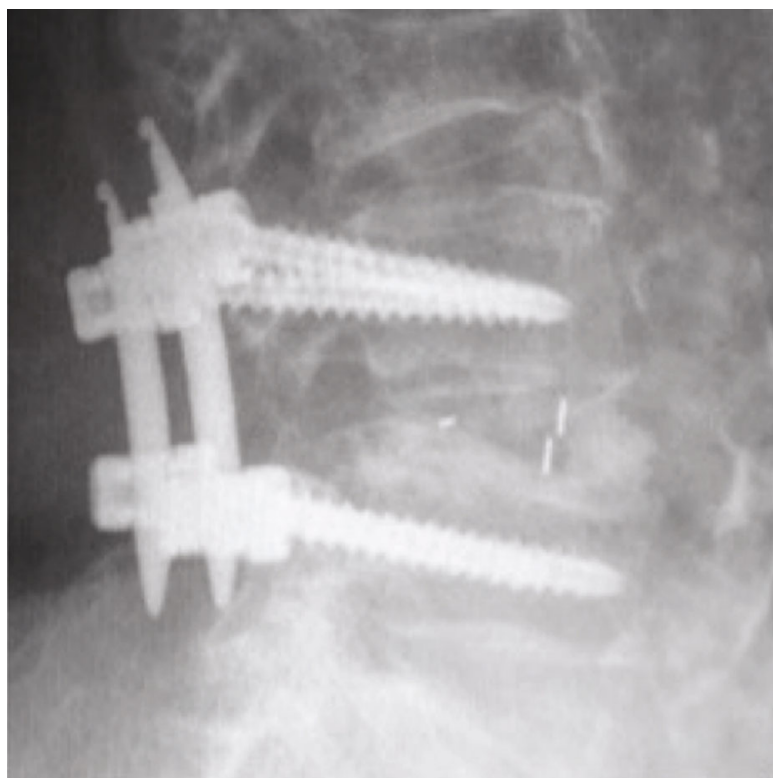

(c)

FIGURE 4: A case showing cage with postoperative subsidence: (a) preoperative lateral radiograph; (b) immediately postoperative radiograph; (c) lateral radiograph of one month after surgery showing cage subsidence.

comparing static and expandable cages concluded that there was no difference in the overall recovery of sagittal alignment. In our study, we also found that MIS-TLIF with expandable cage did not significantly improve SL or LL in patients with osteoporosis compared with static cage.

Although several authors have described that expandable cages increase the risk of intraoperative settlement [22, 23], we found that the use of expandable cages may help to reduce the risk of intraoperative settlement in patients with osteoporosis. The endplate is a cortical bone covering the upper and lower surfaces of the vertebral body. Lower BMD or osteoporosis $T$-score significantly promoted intraoperative endplate injury. Intraoperative endplate injury is an intraoperative complication, which may lead to the gradual sinking of the intervertebral cage into the vertebral body and eventually lead to indirect decompression and bone fusion failure [24, 25]. Most static cages are designed to distract themselves through "warheads." One of the biggest challenges in placing fixed frames through MIS-TLIF is that the aperture of optical disc space is usually very narrow. In patients with osteoporosis, cage implantation and excessive tension may lead to endplate injury. The expandable holder is placed in the disc space in a flat form. This new device can be easily placed through the narrow posterior intervertebral disc space hole and then expanded in the head tail plane. In addition, the cage orientation on the sagittal plane can be 
TABLE 4

\begin{tabular}{lccc}
\hline Factor & Static group $(N=178)$ & Expandable group $(N=106)$ & $P$ value \\
\hline Medium change in SL & $2.58 \pm 1.03$ & $1.91 \pm 0.90$ & 0.213 \\
Medium change in LL & $4.53 \pm 1.61$ & $3.54 \pm 1.44$ & 0.088 \\
Preoperative DH & $6.01 \pm 1.26$ & $6.12 \pm 1.20$ & 0.468 \\
RCD & $1.62 \pm 0.28$ & $1.28 \pm 0.11$ & \\
Complications & & 3 & \\
Durotomy & 4 & 4 & $0.01^{*}$ \\
Screw loosening & 3 & 6 & 0.901 \\
Pseudoarthrosis & 7 & 5 & 0.946 \\
Intraoperative subsidence & 28 & 1 & $0.011^{*}$ \\
Postoperative subsidence & 22 & 5 & $0.034^{*}$ \\
Cage migration & 10 & $0.048^{*}$ \\
Adjacent segment disease & 8 & 0.931 \\
\hline
\end{tabular}

Comparison of radiographic outcomes and complications between the expandable cage group and static cage group. ${ }^{*}$ Statistically significant difference.

adjusted until the cage is parallel to the endplate before expansion. It allows to optimize endplate to endplate assembly while minimizing excessive dispersion. These operating characteristics reduce the risk of endplate damage due to stent insertion and stretch.

An increase in the height of the cage may cause the cage to sink. A cage more than the disc height may increase the axial stress at the cage endplate interface, resulting in immediate or further sinking of the cage. Singhatanadgige et al. [26] found that cage height $\geq 12 \mathrm{~mm}$ was associated with an increased risk of cage sinking. Pisano et al. [27] showed that excessive dispersion of disc height may lead to more cage sinking. Truumees et al. [28] advocated avoiding excessive dispersion. However, due to differences between patients, the definition of "end" remains unclear. In our study, patients with excessive dispersion of the intervertebral space during surgery may have an increased risk of subsidence, manifested as RCD values. We found that RCD was significantly associated with intraoperative and postoperative cage subsidence. For patients with reduced bone mineral density, choosing an appropriate height of interbody fusion cage is the key stage of lumbar interbody fusion, which has an important impact on the clinical effect. However, the appropriate size is difficult to determine. For a long time, surgeons mainly determine it according to their surgical experience. Most surgeons prefer to use a higher interbody fusion cage to restore lumbar alignment and improve the effect of indirect decompression. When using a tractor or cage test, a large impact force may cause endplate injury, especially when the intervertebral space is narrow and the instrument insertion direction is not parallel to the intervertebral space direction. The intraoperative sedimentation rate increased significantly [29]. If too large interbody fusion cage is inserted without damaging the endplate, it will still lead to excessive stretching of the intervertebral space, which may increase the abnormal stress between the endplates and increase the incidence of postoperative subsidence. In our study, the RCD of the static cage group was significantly better than that of the expandable cage group. The size of the distractor and cage test is increased in order to disperse the intervertebral space. The static cage height is mainly determined by the size of the last insertion of the distractor or cage test. We speculate that this is related to increasing the height of the static cage. Unlike the static cage, the expansion degree of the expandable cage depends more on the torsional resistance transmitted by the rotating handle to the surgeon. Due to osteoporosis, the final expansion often ends with less torsion resistance, resulting in less recovery of intervertebral height. Compared with cage height, we believe that RCD can truly reflect the degree of distraction caused by individual differences. Lower RCD in the expandable group may reduce the risk of cage sinking.

3.1. Limitations. The obvious limitation of this study is the retrospective design. Retrospective studies focusing on surgical techniques also introduced inherent surgeon-specific bias and selection bias, adding confounding variables to the analysis. In addition, many cases were excluded due to insufficient data description, which may lead to selection bias. Cage subsidence and displacement were mainly evaluated by plain film rather than CT scan. This may reduce the accuracy of radiographic measurements. Future research will benefit from the increase of CT analysis.

\section{Conclusion}

The use of expandable cages in MIS-TLIF has shown good results in the treatment of degenerative lumbar diseases with osteoporosis. Through appropriate surgical techniques, the expandable cage can reduce the risk of cage sinking.

\section{Data Availability}

No data were used to support this study.

\section{Conflicts of Interest}

The authors declare that they have no conflicts of interest. 


\section{Authors' Contributions}

Yao Li and Shengfu Liu contributed equally to the manuscript and should be considered co-first authors.

\section{References}

[1] D. K. Chin, J. Y. Park, Y. S. Yoon et al., "Prevalence of osteoporosis in patients requiring spine surgery: incidence and significance of osteoporosis in spine disease," Osteoporosis International, vol. 18, pp. 1219-1224, 2007.

[2] H. Kimura, J. Shikata, S. Odate, T. Soeda, and S. Yamamura, "Risk factors for cage retropulsion after posterior lumbar interbody fusion: analysis of 1070 cases," Spine, vol. 37, no. 13, pp. 1164-1169, 2012.

[3] D. Y. Lee, Y. J. Park, S. Y. Song, S. T. Jeong, and D. H. Kim, "Risk factors for posterior cage migration after lumbar interbody fusion surgery," Asian Spine Journal, vol. 12, pp. 59-68, 2018.

[4] A. T. Villavicencio, S. Burneikiene, C. M. Roeca, E. L. Nelson, and A. Mason, "Minimally invasive versus open transforaminal lumbar interbody fusion," Surgical Neurology International, vol. 1, p. 12, 2010.

[5] A. G. Tohmeh, D. Khorsand, B. Watson, and X. Zielinski, "Radiographical and clinical evaluation of extreme lateral interbody fusion: effects of cage size and instrumentation type with a minimum of 1-year follow-up," Spine, vol. 39, no. 26, pp. E1582-E1591, 2014.

[6] D. W. Kucharzyk and L. E. Miller, "Two-year clinical and radiographic results with a multidimensional, expandable interbody implant in minimally invasive lumbar spine surgery," Cureus, vol. 12, article e7070, 2020.

[7] L. T. Holly, J. D. Schwender, D. P. Rouben, and K. T. Foley, "Minimally invasive transforaminal lumbar interbody fusion: indications, technique, and complications," Neurosurgical Focus, vol. 20, pp. 1-5, 2006.

[8] L. T. Khoo, S. Palmer, D. T. Laich, and R. G. Fessler, "Minimally invasive percutaneous posterior lumbar interbody fusion," Neurosurgery, vol. 51, suppl_2, pp. S2-116, 2002.

[9] M. Charlson, T. P. Szatrowski, J. Peterson, and J. Gold, "Validation of a combined comorbidity index," Journal of Clinical Epidemiology, vol. 47, pp. 1245-1251, 1994.

[10] D. H. Ge, N. D. Stekas, C. G. Varlotta et al., "Comparative analysis of two transforaminal lumbar interbody fusion techniques: open TLIF versus Wiltse MIS TLIF," Spine, vol. 44, no. 9, pp. E555-E560, 2019.

[11] N. R. Khan, A. J. Clark, S. L. Lee, G. T. Venable, N. B. Rossi, and K. T. Foley, "Surgical outcomes for minimally invasive vs open transforaminal lumbar interbody fusion: an updated systematic review and meta-analysis," Neurosurgery, vol. 77, pp. 847-874, 2015.

[12] S. Fukuta, K. Miyamoto, H. Hosoe, and K. Shimizu, "Kidneytype intervertebral spacers should be located anteriorly in cantilever transforaminal lumbar interbody fusion: analyses of risk factors for spacer subsidence for a minimum of 2 years," Journal of Spinal Disorders \& Techniques, vol. 24, pp. 189-195, 2011.

[13] Y. Hou and W. Yuan, "Influences of disc degeneration and bone mineral density on the structural properties of lumbar end plates," The Spine Journal, vol. 12, pp. 249-256, 2012.

[14] C. Jones, I. Okano, S. N. Salzmann et al., "Endplate volumetric bone mineral density is a predictor for cage subsidence following lateral lumbar interbody fusion: a risk factor analysis," The Spine Journal, vol. 21, pp. 1729-1737, 2021.
[15] K. W. Oh, J. H. Lee, J. H. Lee, D. Y. Lee, and H. J. Shim, “The correlation between cage subsidence, bone mineral density, and clinical results in posterior lumbar interbody fusion," Clinical Spine Surgery, vol. 30, pp. E683-E689, 2017.

[16] J. H. Cho, C. J. Hwang, H. Kim, Y. S. Joo, D. H. Lee, and C. S. Lee, "Effect of osteoporosis on the clinical and radiological outcomes following one-level posterior lumbar interbody fusion," Journal of Orthopaedic Science, vol. 23, pp. 870-877, 2018.

[17] T. J. Yee, J. R. Joseph, S. W. Terman, and P. Park, "Expandable vs static cages in transforaminal lumbar interbody fusion: radiographic comparison of segmental and lumbar sagittal angles," Neurosurgery, vol. 81, pp. 69-74, 2017.

[18] M. Alimi, B. Shin, M. Macielak et al., "Expandable polyarylether-ether-ketone spacers for interbody distraction in the lumbar spine," Global Spine Journal, vol. 5, pp. 169-178, 2015.

[19] C. W. Kim, T. M. Doerr, I. Y. Luna et al., "Minimally invasive transforaminal lumbar interbody fusion using expandable technology: a clinical and radiographic analysis of 50 patients," World Neurosurgery, vol. 90, pp. 228-235, 2016.

[20] L. W. Massie, H. M. Zakaria, L. R. Schultz, A. Basheer, M. A. Buraimoh, and V. Chang, "Assessment of radiographic and clinical outcomes of an articulating expandable interbody cage in minimally invasive transforaminal lumbar interbody fusion for spondylolisthesis,” Neurosurgical Focus, vol. 44, p. E8, 2018.

[21] J. H. McMordie, K. P. Schmidt, A. P. Gard, and C. C. Gillis, "Clinical and short-term radiographic outcomes of minimally invasive transforaminal lumbar interbody fusion with expandable lordotic devices," Neurosurgery, vol. 86, pp. E147-E155, 2020.

[22] C. C. Chang, D. Chou, B. Pennicooke et al., "Long-term radiographic outcomes of expandable versus static cages in transforaminal lumbar interbody fusion," Journal of Neurosurgery. Spine, vol. 34, pp. 1-10, 2020.

[23] C. Stickley, T. Philipp, E. Wang et al., "Expandable cages increase the risk of intraoperative subsidence but do not improve perioperative outcomes in single level transforaminal lumbar interbody fusion," The Spine Journal, vol. 21, pp. 3744, 2021.

[24] Y. Hou and Z. Luo, "A study on the structural properties of the lumbar endplate: histological structure, the effect of bone density, and spinal level," Spine, vol. 34, no. 12, pp. E427-E433, 2009.

[25] K. Satake, T. Kanemura, H. Yamaguchi, N. Segi, and J. Ouchida, "Predisposing factors for intraoperative endplate injury of extreme lateral interbody fusion," Asian Spine Journal, vol. 10, pp. 907-914, 2016.

[26] W. Singhatanadgige, A. Sukthuayat, T. Tanaviriyachai et al., "Risk factors for polyetheretherketone cage subsidence following minimally invasive transforaminal lumbar interbody fusion," Acta Neurochirurgica, vol. 163, pp. 2557-2565, 2021.

[27] A. J. Pisano, D. R. Fredericks, T. Steelman, C. Riccio, M. D. Helgeson, and S. C. Wagner, "Lumbar disc height and vertebral Hounsfield units: association with interbody cage subsidence," Neurosurgical Focus, vol. 49, p. E9, 2020.

[28] E. Truumees, C. K. Demetropoulos, K. H. Yang, and H. N. Herkowitz, "Effects of disc height and distractive forces on graft compression in an anterior cervical discectomy model," Spine, vol. 27, no. 22, pp. 2441-2445, 2002.

[29] N. Li, M. Dai, B. Zhang et al., "Risk factors for cage retropulsion after transforaminal lumbar interbody fusion in older patients," Annals of Translational Medicine, vol. 8, p. 1660, 2020. 